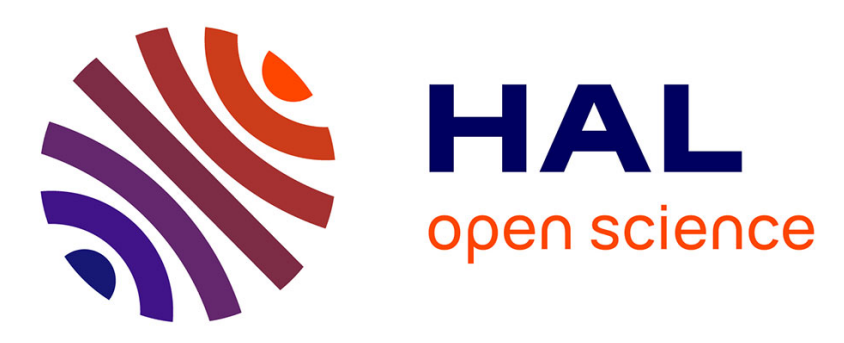

\title{
A novel control scheme for teleoperation with guaranteed performance under time-varying delays
}

Bo Zhang, Alexandre Kruszewski, Jean-Pierre Richard

\section{To cite this version:}

Bo Zhang, Alexandre Kruszewski, Jean-Pierre Richard. A novel control scheme for teleoperation with guaranteed performance under time-varying delays. 23th Chinese Control and Decision Conference (CCDC), Jun 2011, Mianyang, China. hal-00602338

\section{HAL Id: hal-00602338 \\ https://hal.science/hal-00602338}

Submitted on 22 Jun 2011

HAL is a multi-disciplinary open access archive for the deposit and dissemination of scientific research documents, whether they are published or not. The documents may come from teaching and research institutions in France or abroad, or from public or private research centers.
L'archive ouverte pluridisciplinaire HAL, est destinée au dépôt et à la diffusion de documents scientifiques de niveau recherche, publiés ou non, émanant des établissements d'enseignement et de recherche français ou étrangers, des laboratoires publics ou privés. 


\title{
A Novel Control Scheme for Teleoperation with Guaranteed Performance under Time-Varying Delays
}

\author{
Bo Zhang, Alexandre Kruszewski and Jean-Pierre Richard
}

\begin{abstract}
This work deals with the stability and synchronization of systems with time-varying delays. We propose a novel control scheme with position/velocity information channel on the basis of Lyapunov-Krasovskii functional (LKF) and $H_{\infty}$ control theory by using Linear Matrix Inequality (LMI). The proposed solution is efficient for different working conditions, such as abrupt motion and wall contact, and this is illustrated by various simulations.

Index Terms-Teleoperation System, Time-Varying Delay, $H_{\infty}$ Control, Lyapunov-Krasovskii Functional, Linear Matrix Inequality
\end{abstract}

\section{INTRODUCTION}

The concept of teleoperation implies a dual system, in which a remote slave robot tracks the motion of a master manipulator. It must include a communication medium, so that the position/velocity information of the master manipulator handled by the human operator is delivered to the slave robot, and the corresponding data of the slave is transmitted back to the master. It constitutes a Networked Control System in which the communication channels, especially the Internet, introduce additional dynamics represented by time-varying delays [11] [15]. In order to avoid a severe deterioration of the global performance, these delays must be considered at the control design stage [1] [2].

The passivity formalism represents the most popular approach for Velocity-Force (VF) schemes in teleoperation. Since the cornerstone papers of Anderson and Spong [1], Niemeyer and Slotine [10], the passivity, scattering and wave variables allow for including arbitrary time delays into systems in a passive and hence stable fashion. Besides, another formulation is the passivity-based structure without the transformation of wave variables. A recent approach is the energy based time domain passivity control (ETDPC) [12]. Overall, passivity-based approaches can deal with stabilization and velocity tracking under any time-varying delays. But, as it was already noted in [12], passivity does not allow for optimizing the system performance, which keeps decreasing as the communication delays increase and does not guarantee the position tracking in general.

Thus, from the point of view of performance, it is desirable to design a controller ensuring the position tracking with prescribed convergence rate, which of course will be linked to the Quality of Service available from the network. Various control strategies have been proposed in this area. In the

\{Bo Zhang, Alexandre Kruszewski and Jean-Pierre Richard\} are with Université Lille Nord de France, Ecole Centrale de Lille - 59651 Villeneuve d'Ascq, France and LAGIS, CNRS FRE3303, Laboratoire d'Automatique, Génie Informatique et Signal. J.P.Richard is also with INRIA NON_A. case of a constant communication delay, Chopra et al. [3] proposed a new system configuration for bilateral teleoperation in order to guarantee the position tracking. Later on, Garcia-Valdovinos and Parra-Vega [6] designed a new observer-based higher-order sliding mode impedance control strategy.

The present paper aims at considering the case of variable delays. With this aim in mind, Lyapunov approaches for time-delay systems are helpful [11]. We will both ensure the stability of the teleoperation system and realize high $H_{\infty}$ performance of the position tracking. In the case of timevarying delays, many stability conditions have been proposed in terms of Lyapunov-Krasovskii functionals (LKF), which can be solved by Linear Matrix Inequality (LMI). Valuable results can be found in the paper [4] by Fridman (see also the included references). For $H_{\infty}$ performance consideration, we will also use results from [4] [5] on the $H_{\infty}$ control of systems with delays (see also [14] [16] for $H_{\infty}$ control with time-varying delays). These two approaches will be helpful to design a novel teleoperation system scheme, which makes use of LKF to ensure the stability, and further, realize the position tracking by $H_{\infty}$ control.

This paper is organized as follows: Section 2 introduces and briefly explains the theorems to be used later. The problem under consideration is briefly presented in section 3. In section 4, the bilateral teleoperation system using the LKF and state-feedback $H_{\infty}$ control is given. Results of simulation are presented in section 5. Finally we conclude and discuss the future work in section 6 .

\section{PRELIMINARIES}

In the next section, the teleoperation system will be modeled as a linear time-varying delay system. This section is devoted to the stability and the performance analysis of this class of models, which is described by,

$$
\left(\Sigma_{1}\right)\left\{\begin{array}{l}
\dot{x}(t)=\sum_{i=0}^{n} A_{i} x\left(t-\tau_{i}(t)\right) \\
x\left(t_{0}+\theta\right)=\phi(\theta), \dot{x}\left(t_{0}+\theta\right)=\dot{\phi}(\theta), \theta \in\left[-h_{2}, 0\right]
\end{array}\right.
$$

where, $x(t) \in \mathbf{R}^{n}$ is the state, $\tau_{0}(t) \equiv 0, \phi(\theta)$ is the initial condition, and the time-varying delays, $\tau_{i}(t) \in\left[h_{1}, h_{2}\right]$, $h_{1} \geq 0, i=1,2, \ldots, n$. Considering the following LyapunovKrasovskii functional [4], 


$$
\begin{aligned}
& V(t, x(t), \dot{x}(t))=x(t)^{T} P x(t) \\
& +\int_{t-h_{2}}^{t} x(s)^{T} S_{a} x(s) d s+\int_{t-h_{1}}^{t} x(s)^{T} S x(s) d s \\
& +h_{1} \int_{-h_{1}}^{0} \int_{t+\theta}^{t} \dot{x}(s)^{T} R \dot{x}(s) d s d \theta \\
& +\sum_{i=1}^{n}\left(h_{2}-h_{1}\right) \int_{-h_{2}}^{-h_{1}} \int_{t+\theta}^{t} \dot{x}(s)^{T} R_{a i} \dot{x}(s) d s d \theta
\end{aligned}
$$

Theorem 1: Suppose there exists $n \times n$ matrices $P>0$, $R>0, S>0, S_{a}>0, R_{a i}>0, P_{2}, P_{3}, Y_{1}, Y_{2}, i=$ $1,2, \ldots, n$, such that the condition (3) with notations (4) at the top of next page is feasible, the system (1) is asymptotically stable for time-varying delays $\tau_{i}(t) \in\left[h_{1}, h_{2}\right], i=1,2, \ldots, n$.

Proof. The theorem is the extension of [9] and the proof is straightforward.

Based on Theorem 1, the Lyapunov-Krasovskii functional stability condition with several time-varying delays is used to derive LMI stability conditions, which can be solved efficiently. Further, this paper is not only concentrated in the guarantee of stability, but also in the improvement of the overall performances under time-varying delays, so we employ Bounded Real Lemma (BRL) based on Theorem 1 [5].

Generally, given the following system,

$$
\left(\Sigma_{2}\right)\left\{\begin{array}{l}
\dot{x}(t)=\sum_{i=0}^{n} A_{i} x\left(t-\tau_{i}(t)\right)+B w(t) \\
z(t)=C x(t)
\end{array}\right.
$$

where, new term $w(t) \in \mathbf{R}^{l}$ is defined as the exogenous disturbance signal, and $z(t) \in \mathbf{R}^{q}$ is seen as the objective control output, $C$ is a constant matrice.

For a prescribed scalar $\gamma$, we define the performance index,

$$
J(w)=\int_{0}^{\infty}\left(z(t)^{T} z(t)-\gamma^{2} w(t)^{T} w(t)\right) d t
$$

Then, according to the theory of $H_{\infty}$ control, we can ensure the stability and optimize the performance of the system with time-varying delays by verifying the performance index,

$$
J(w)<0
$$

So, we obtain the following Theorem as follow,

Theorem 2: Suppose there exists $n \times n$ matrices $P>0$, $R>0, S>0, S_{a}>0, R_{a i}>0, P_{2}, P_{3}, Y_{1}, Y_{2}, i=$ $1,2, \ldots, n$, and a positive scale $\gamma$, such that the condition (8) with notations (9) at the top of next page is feasible, the system (5) is asymptotically stable and $J(w)<0$ for timevarying delays $\tau_{i}(t) \in\left[h_{1}, h_{2}\right], i=1,2, \ldots, n$.

Proof. To ensure $J(w)<0$, we consider the condition,

$$
\dot{V}(t, x(t), \dot{x}(t))+z(t)^{T} z(t)-\gamma^{2} w(t)^{T} w(t)<0
$$

Integrating the resulting inequality in $t$ from 0 to $\infty$,

$$
\begin{aligned}
& \int_{0}^{\infty}\left(\dot{V}(t, x(t), \dot{x}(t))+z(t)^{T} z(t)-\gamma^{2} w(t)^{T} w(t)\right) d t \\
& =V(\infty, x(\infty), \dot{x}(\infty))-V(0, x(0), \dot{x}(0)) \\
& +\int_{0}^{\infty}\left(z(t)^{T} z(t)-\gamma^{2} w(t)^{T} w(t)\right) d t \\
& <0
\end{aligned}
$$

Because $V(0, x(0), \dot{x}(0))=0$ and $V(\infty, x(\infty), \dot{x}(\infty)) \geq$ 0 , we can assure $J(w)<0$ by adding the term $z(t)^{T} z(t)-$ $\gamma^{2} w(t)^{T} w(t)$ into $\dot{V}(t, x(t), \dot{x}(t))$. Considering the system of (14), we get,

$$
\begin{aligned}
& \dot{V}(t, x(t), \dot{x}(t))+z(t)^{T} z(t)-\gamma^{2} w(t)^{T} w(t) \\
& =x(t)^{T}\left(S+S_{a}\right) x(t) \\
& +\dot{x}(t)^{T} P x(t)+x(t)^{T} P \dot{x}(t) \\
& -x\left(t-h_{1}\right)^{T} S x\left(t-h_{1}\right) \\
& -x\left(t-h_{2}\right)^{T} S_{a} x\left(t-h_{2}\right) \\
& +\dot{x}(t)^{T}\left[h_{1}^{2} R+\left(h_{2}-h_{1}\right)^{2} \sum_{i=1}^{n} R_{a i}\right] \dot{x}(t) \\
& -h_{1} \int_{t-h_{1}}^{t} \dot{x}(s)^{T} R \dot{x}(s) d s \\
& -\left(h_{2}-h_{1}\right) \int_{t-h_{2}}^{t-h_{1}} \dot{x}(s)^{T} \sum_{i=1}^{n} R_{a i} \dot{x}(s) d s \\
& +z(t)^{T} z(t)-\gamma^{2} w(t)^{T} w(t)
\end{aligned}
$$

Then, substituting for $z(t)$ and $w(t)$, the derivation process is same as for Theorem 1. Applying the Jensen's inequality [7], then obtain,

$$
\begin{aligned}
& \dot{V}(t, x(t), \dot{x}(t))+z(t)^{T} z(t)-\gamma^{2} w(t)^{T} w(t) \\
& \leq x(t)^{T}\left(S+S_{a}+C^{T} C\right) x(t)+\dot{x}(t)^{T} P x(t)+x(t)^{T} P \dot{x}(t) \\
& -x\left(t-h_{1}\right)^{T} S x\left(t-h_{1}\right)-x\left(t-h_{2}\right)^{T} S_{a} x\left(t-h_{2}\right) \\
& +\dot{x}(t)^{T}\left[h_{1}^{2} R+\left(h_{2}-h_{1}\right)^{2} \sum_{i=1}^{n} R_{a i}\right] \dot{x}(t) \\
& -\left[x(t)^{T}-x\left(t-h_{1}\right)^{T}\right] R\left[x(t)-x\left(t-h_{1}\right)\right] \\
& -\sum_{i=1}^{n} v_{1 i}^{T} R_{a i} v_{1 i}-\sum_{i=1}^{n} v_{2 i}^{T} R_{a i} v_{2 i} \\
& -w(t)^{T} \gamma^{2} I_{l} w(t)
\end{aligned}
$$

where,

$$
\begin{aligned}
v_{1 i} & =\int_{t-\tau_{i}(t)}^{t-h_{1}} \dot{x}(s) d s \\
v_{2 i} & =\int_{t-h_{2}}^{t-\tau_{i}(t)} \dot{x}(s) d s, \quad i=1,2, \ldots, n
\end{aligned}
$$

By using the descriptor method and free weighting matrices [4] [8], for some $n \times n$ matrices $P_{2}, P_{3}, Y_{1}, Y_{2}$, the expression as follows is added into $\dot{V}(t, x(t), \dot{x}(t))+$ $z(t)^{T} z(t)-\gamma^{2} w(t)^{T} w(t)$ 


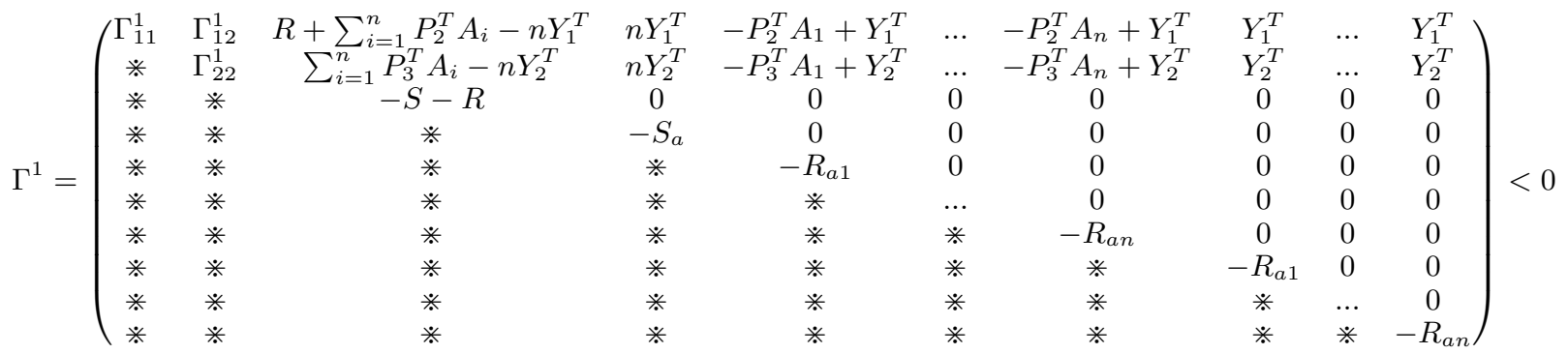

$$
\begin{aligned}
& \Gamma_{11}^{1}=S+S_{a}-R+A_{0}^{T} P_{2}+P_{2}^{T} A_{0}, \quad \Gamma_{12}^{1}=P-P_{2}^{T}+A_{0}^{T} P_{3}, \quad \Gamma_{22}^{1}=-P_{3}-P_{3}^{T}+h_{1}^{2} R+\left(h_{2}-h_{1}\right)^{2} \sum_{i=1}^{n} R_{a i}
\end{aligned}
$$

$\Gamma^{2}=\left(\begin{array}{ccc}\Gamma_{11}^{2} & \Gamma_{12}^{2} & R+\sum_{i=1}^{n} P_{2}^{T} A_{i}-n Y_{1}^{T} \\ * & \Gamma_{22}^{2} & \sum_{i=1}^{n} P_{3}^{T} A_{i}-n Y_{2}^{T} \\ * & * & -S-R \\ * & * & * \\ * & * & * \\ * & * & * \\ * & * & * \\ * & * & * \\ * & * & * \\ * & * & *\end{array}\right.$

$\begin{array}{cc}n Y_{1}^{T} & -P_{2}^{T} A_{1}+Y_{1}^{T} \\ n Y_{2}^{T} & -P_{3}^{T} A_{1}+Y_{2}^{T} \\ 0 & 0 \\ -S_{a} & 0 \\ * & -R_{a 1} \\ * & * \\ * & * \\ * & * \\ * & * \\ * & * \\ * & *\end{array}$

$\begin{array}{cc}\ldots & -P_{2}^{T} A_{n}+Y_{1}^{T} \\ \ldots & -P_{3}^{T} A_{n}+Y_{2}^{T} \\ 0 & 0 \\ 0 & 0 \\ 0 & 0 \\ \ldots & 0 \\ * & -R_{a n} \\ * & * \\ * & * \\ * & * \\ * & *\end{array}$

$$
\left.\begin{array}{cccc}
Y_{1}^{T} & \ldots & Y_{1}^{T} & P_{2}^{T} B \\
Y_{2}^{T} & \ldots & Y_{2}^{T} & P_{3}^{T} B \\
0 & 0 & 0 & 0 \\
0 & 0 & 0 & 0 \\
0 & 0 & 0 & 0 \\
0 & 0 & 0 & 0 \\
0 & 0 & 0 & 0 \\
-R_{a 1} & 0 & 0 & 0 \\
* & \cdots & 0 & 0 \\
* & * & -R_{a n} & 0 \\
* & * & * & -\gamma^{2} I
\end{array}\right)<0
$$

$\Gamma_{11}^{2}=S+S_{a}-R+A_{0}^{T} P_{2}+P_{2}^{T} A_{0}+C^{T} C, \quad \Gamma_{12}^{2}=P-P_{2}^{T}+A_{0}^{T} P_{3}, \quad \Gamma_{22}^{2}=-P_{3}-P_{3}^{T}+h_{1}^{2} R+\left(h_{2}-h_{1}\right)^{2} \sum_{i=1}^{n} R_{a i}$

$$
\begin{aligned}
0= & 2\left[x(t)^{T} P_{2}^{T}+\dot{x}(t)^{T} P_{3}^{T}\right] \\
& {\left[A_{0} x(t)+B w(t)+\sum_{i=1}^{n} A_{i} x\left(t-h_{1}\right)-\sum_{i=1}^{n} A_{i} v_{1 i}-\dot{x}(t)\right] } \\
0= & 2\left[x(t)^{T} Y_{1}^{T}+\dot{x}(t)^{T} Y_{2}^{T}\right] \\
& {\left[n x\left(t-h_{2}\right)+\sum_{i=1}^{n} v_{1 i}+\sum_{i=1}^{n} v_{2 i}-n x\left(t-h_{1}\right)\right] }
\end{aligned}
$$

Setting,

$$
\begin{aligned}
\eta(t)= & \operatorname{col}\left\{x(t), \dot{x}(t), x\left(t-h_{1}\right), x\left(t-h_{2}\right),\right. \\
& \left.v_{11}, v_{12}, \ldots, v_{1 n}, v_{21}, v_{22}, \ldots, v_{2 n}, w(t)\right\}
\end{aligned}
$$

Finally, if the LMI in (8) is feasible, we obtain,

$$
\begin{aligned}
& \dot{V}(t, x(t), \dot{x}(t))+z(t)^{T} z(t)-\gamma^{2} w(t)^{T} w(t) \\
& \leq \eta(t)^{T} \Gamma \eta(t)<0
\end{aligned}
$$

Note that if (8) holds, then the LMI (3) is feasible, thus the system (5) is asymptotically stable under the $H_{\infty}$ constraint.

\section{PROBLEM STATEMENT}

A teleoperation system is composed of five entities: the Human Operator, the Haptic Interface (Master), the Remote Robot (Slave), the Environment and the Communication Network. The master and the slave are actuated mechanical systems with the same number $N$ of DOF. All these entities are connected according to the novel scheme given in Fig. 1, where $F_{m}(t)$ and $F_{s}(t)$ are the actuated inputs of the master and the slave, $F_{h}(t)$ and $F_{e}(t)$ are the effects of the human operator and environment on the system, $C_{1}$ and $C_{2}$ are the controllers, $\tau_{1}(t)$ and $\tau_{2}(t)$ are the network delays. $x_{m}(t)$ and $x_{s}(t)$ are the state of the master and the slave, they are composed with the speed vectors $\left(\dot{\theta}_{m}(t)\right.$ and $\left.\dot{\theta}_{s}(t)\right)$ and the position vectors $\left(\theta_{m}(t)\right.$ and $\left.\theta_{s}(t)\right)$.

The following assumptions are made,

1) The master and the slave are linear dynamical systems.

2) The communication delays are bounded.

3) The data packet exchanged between the master and the slave are time-stamped and the master and the slave clock are synchronized.

4) The master and the slave systems have a local controller ensuring the marginal stability (only the speed stability).

The goal is to design the controllers $C_{1}$ and $C_{2}$ on each side of the network ensuring the bilateral position tracking of the master and the slave under communication delays. To reach this goal, the human operator and the environment are considered as an unknown inputs and the controllers have to minimize their effects on the output tracking.

The expression of the controllers $C_{1}$ and $C_{2}$ are given by, 


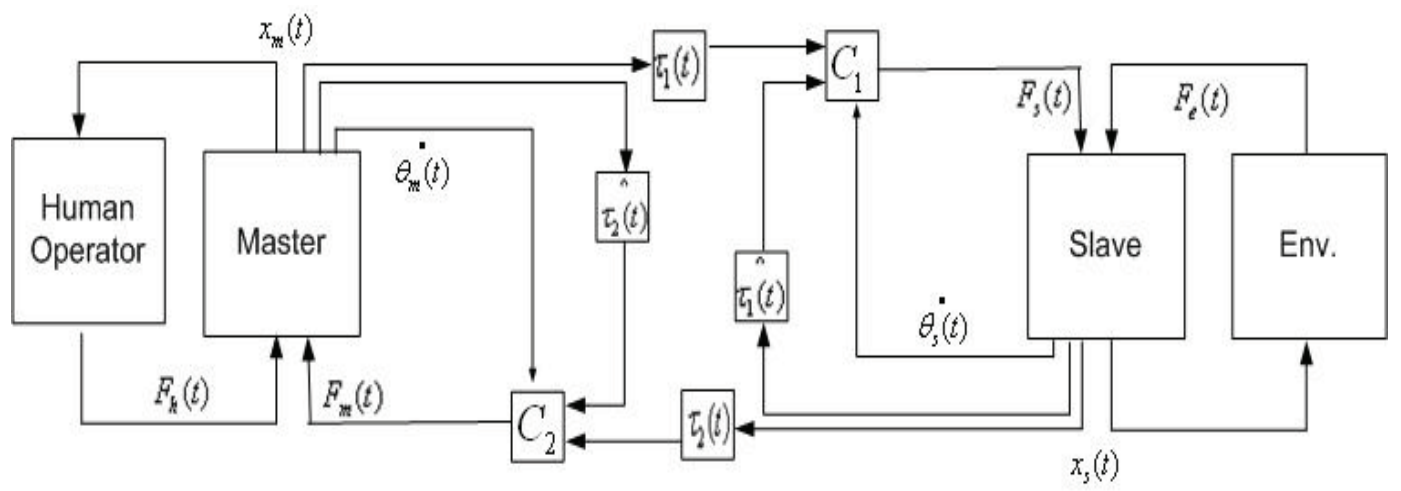

Fig. 1. Novel Teleoperation Control Scheme

$$
\begin{aligned}
F_{s}(t)= & -K_{0}^{s} \dot{\theta}_{s}(t)-K_{1}^{1} \dot{\theta}_{s}\left(t-\hat{\tau}_{1}(t)\right)-K_{1}^{2} \dot{\theta}_{m}\left(t-\tau_{1}(t)\right) \\
& -K_{1}^{3}\left(\theta_{s}\left(t-\hat{\tau}_{1}(t)-\theta_{m}\left(t-\tau_{1}(t)\right)\right)\right. \\
F_{m}(t)= & -K_{0}^{m} \dot{\theta}_{m}(t)-K_{2}^{1} \dot{\theta}_{s}\left(t-\tau_{2}(t)\right)-K_{2}^{2} \dot{\theta}_{m}\left(t-\hat{\tau}_{2}(t)\right) \\
& -K_{2}^{3}\left(\theta_{s}\left(t-\tau_{2}(t)-\theta_{m}\left(t-\hat{\tau}_{2}(t)\right)\right)\right.
\end{aligned}
$$

where the control gains $\left(K_{0}^{s}\right.$ and $\left.K_{0}^{m}\right)$ are local partial state feedbacks. They are supposed to be known according to the assumption 4. $\hat{\tau_{1}}(t)$ and $\hat{\tau_{2}}(t)$ are estimated network delays between master and slave. Because of assumption 3, the delays can be measured: $\hat{\tau_{1}}(t)=\tau_{1}(t), \hat{\tau_{2}}(t)=\tau_{2}(t)$. The control gains $K_{i}^{j}, i=1,2, j=1,2,3$, are the ones to be designed for the bilateral teleoperation.

Taking into account all the assumptions made, we can describe the teleoperation problem as the stabilization of the following linear system,

$$
\left(\Sigma_{3}\right)\left\{\begin{aligned}
\dot{x}(t) & =A x(t)+B u(t)+B w(t) \\
u(t) & =-K_{0} x(t)-K_{1} x\left(t-\tau_{1}(t)\right) \\
& -K_{2} x\left(t-\tau_{2}(t)\right)
\end{aligned}\right.
$$

where, $x(t), u(t)$ are respectively the state and input of the complete system, which involve the master and slave in general. The detailed explanation of the system state and the input,

$$
\begin{aligned}
& x(t)=\operatorname{col}\left\{\dot{\theta}_{s}(t), \dot{\theta}_{m}(t), \theta_{s}(t)-\theta_{m}(t)\right\} \\
& u(t)=\operatorname{col}\left\{F_{s}(t), F_{m}(t)\right\}, \quad w(t)=\operatorname{col}\left\{F_{e}(t), F_{h}(t)\right\}
\end{aligned}
$$

where, $i=1,2$,

$$
K_{0}=\left[\begin{array}{ccc}
K_{0}^{s} & 0 & 0 \\
0 & K_{0}^{m} & 0
\end{array}\right], \quad K_{i}=\left[\begin{array}{lll}
K_{i}^{1} & K_{i}^{2} & K_{i}^{3}
\end{array}\right]
$$

\section{MAIN RESULTS}

The goal of this section is to provide a control design scheme to achieve the stability of the system with guaranteed performance. The stability conditions are ensured for the global system. Firstly, the effect of the human operator and the environment are neglected (i.e. $w(t)=0$ ). The closedloop system can be written as,
$\left(\Sigma_{4}\right): \dot{x}(t)=A_{0} x(t)+A_{1} x\left(t-\tau_{1}(t)\right)+A_{2} x\left(t-\tau_{2}(t)\right)$

where,

$$
A_{0}=A-B K_{0}, \quad A_{1}=-B K_{1}, \quad A_{2}=-B K_{2}
$$

The following theorem provides LMI conditions allowing the design of a stabilizing controller for the teleoperation problem (i.e. the stability of 22),

Theorem 3: Suppose there exists matrices $P>0, R>0$, $S>0, S_{a}>0, R_{a 1}>0, R_{a 2}>0, P_{2}, W_{1}, W_{2}, Y_{1}$, $Y_{2}$, and a positive scale $\xi$, such that the condition (24) with notations (25) at the top of next page is feasible, the system (22) is asymptotically stable for time-varying delays $\tau_{1}(t)$, $\tau_{2}(t) \in\left[h_{1}, h_{2}\right]$. The control gains are given by,

$$
K_{1}=W_{1} P_{2}^{-1}, \quad K_{2}=W_{2} P_{2}^{-1}
$$

Proof. We substitute the system (22) into Theorem 1, a nonlinear matrix inequality is obtained due to the terms $P_{2}^{T} B K_{1}, P_{2}^{T} B K_{2}, P_{3}^{T} B K_{1}, P_{3}^{T} B K_{2}$.

We choose $P_{3}=\xi P_{2}$, and multiply $\Gamma^{1}$ in Theorem 1 by $\operatorname{diag}\left\{P_{2}^{-T}, \ldots, P_{2}^{-T}\right\}$ at the left side, $\operatorname{diag}\left\{P_{2}^{-1}, \ldots, P_{2}^{-1}\right\}$ at the right side, then we get Theorem 3.

Up to this, in this paper, the stability of teleoperation system has been ensured. Next, on the basis of stability, we need to improve the performance of system by optimizing the tracking of position between the master and slave. So, we will take into account the unknown input of the human operator and the environment $F_{h}(t)$ and $F_{e}(t)($ i.e. $w(t) \neq 0)$ on the base of state-feedback $H_{\infty}$ control.

In this context, the closed-loop system can be rewritten,

$$
\left(\Sigma_{5}\right)\left\{\begin{aligned}
\dot{x}(t) & =A_{0} x(t)+A_{1} x\left(t-\tau_{1}(t)\right) \\
& +A_{2} x\left(t-\tau_{2}(t)\right)+B w(t) \\
z(t) & =C x(t)
\end{aligned}\right.
$$

where the exogenous disturbance signal $w(t)=$ $\operatorname{col}\left\{F_{e}(t), F_{h}(t)\right\}$, the objective control output $z(t)=$ $\theta_{s}(t)-\theta_{m}(t) . C$ is a known matrice. The definition of $A_{0}$, $A_{1}, A_{2}$ can be found in (23). 


$$
\begin{aligned}
& \Gamma^{3}=\left(\begin{array}{cccccccc}
\Gamma_{11}^{3} & \Gamma_{12}^{3} & R-B W_{1}-B W_{2}-2 Y_{1}^{T} & 2 Y_{1}^{T} & Y_{1}^{T}+B W_{1} & Y_{1}^{T}+B W_{2} & Y_{1}^{T} & Y_{1}^{T} \\
* & \Gamma_{22}^{3} & -\xi B W_{1}-\xi B W_{2}-2 Y_{2}^{T} & 2 Y_{2}^{T} & Y_{2}^{T}+\xi B W_{1} & Y_{2}^{T}+\xi B W_{2} & Y_{2}^{T} & Y_{2}^{T} \\
* & * & -S-R & 0 & 0 & 0 & 0 & 0 \\
* & * & * & -S_{a} & 0 & 0 & 0 & 0 \\
* & * & * & * & -R_{a 1} & 0 & 0 & 0 \\
* & * & * & * & * & -R_{a 2} & 0 & 0 \\
* & * & * & * & * & * & -R_{a 1} & 0 \\
* & * & * & * & * & * & * & -R_{a 2}
\end{array}\right)<0 \\
& \Gamma_{11}^{3}=S+S_{a}-R+P_{2}^{T} A^{T}+A P_{2}-B K_{0} P_{2}-P_{2}^{T} K_{0}^{T} B^{T}, \quad \Gamma_{12}^{3}=P-P_{2}+\xi P_{2}^{T} A^{T}-\xi P_{2}^{T} K_{0}^{T} B^{T} \\
& \Gamma_{22}^{3}=-\xi P_{2}-\xi P_{2}^{T}+h_{1}^{2} R+\left(h_{2}-h_{1}\right)^{2}\left(R_{a 1}+R_{a 2}\right)
\end{aligned}
$$

Our objective is to minimize $z(t)$ by using $H_{\infty}$ control theory, that is to minimize the deviation of position between the master and slave, $\theta_{s}(t)-\theta_{m}(t)$. Based on Theorem 2, the following result is proposed which allows the optimization of the $H_{\infty}$ bound in form of LMI,

Theorem 4: Suppose there exists matrices $P>0, R>0$, $S>0, S_{a}>0, R_{a 1}>0, R_{a 2}>0, P_{2}, W_{1}, W_{2}, Y_{1}, Y_{2}$, and positive scales $\gamma$ and $\xi$, such that the condition (29) with notations (30) is feasible, the system (27) is asymptotically stable and $J(w)<0$ for time-varying delays $\tau_{1}(t), \tau_{2}(t) \in$ $\left[h_{1}, h_{2}\right]$. The control gains are given by,

$$
\begin{gathered}
K_{1}=W_{1} P_{2}^{-1}, \quad K_{2}=W_{2} P_{2}^{-1} \\
\Gamma^{4}=\left(\begin{array}{ccc}
\Gamma_{11}^{4} & \Gamma_{12}^{4} & \Gamma_{13}^{4} \\
* & \Gamma_{22}^{4} & 0 \\
* & * & \Gamma_{33}^{4}
\end{array}\right)<0 \\
\Gamma_{11}^{4}=\Gamma^{3}, \quad \Gamma_{12}^{4}=\left(\begin{array}{c}
B \\
\xi B \\
0 \\
\vdots \\
0
\end{array}\right), \quad \Gamma_{13}^{4}=\left(\begin{array}{c}
P_{2}^{T} C^{T} \\
0 \\
\vdots \\
0
\end{array}\right) \\
\Gamma_{22}^{4}=-\gamma^{2} I, \quad \Gamma_{33}^{4}=-I
\end{gathered}
$$

Remark 1: Theorem 4 is an extended application of Theorem 2, $K_{1}$ and $K_{2}$ are fixed by $W_{1}$ and $W_{2}$ under the minimum value of $\gamma_{\text {min }}$.

Remark 2: The $H_{\infty}$ control design objective is to minimize the norm of the closed-loop mapping $w(t) \rightarrow z(t)$. More precisely, we look for a minimum characterization of levels $\gamma$, which is defined as $\gamma_{\min }$, therefore, the bound $\sup _{w}\left(\|z(t)\|_{2} /\|w(t)\|_{2}\right)<\gamma_{\min }$ is achievable in the closed-loop system. Therefore, by Theorem 4, we can minimize the deviation of position $z(t)$, in the condition of exogenous disturbance input $w(t)$, further achieve some minimal level of synchronization, as the tracking of position. Besides, the performance of synchronization is proportional to the magnitude of $\gamma_{\min }$, the smaller $\gamma_{\min }$ produces the better performance of system.

\section{SIMULATION}

To evaluate the performance of the proposed approach of teleoperation system, different working conditions have been simulated in MATLAB/SIMULINK. The maximum amplitude and sampling time of time-varying delays are $0.2 \mathrm{~s}$ and $0.001 s$, which satisfy most network-based applications of teleoperation system, as internet-based teleoperation system. Notice that, the time-varying delays in two channels are not correlated.

\section{A. Tracking in abrupt changing motion}
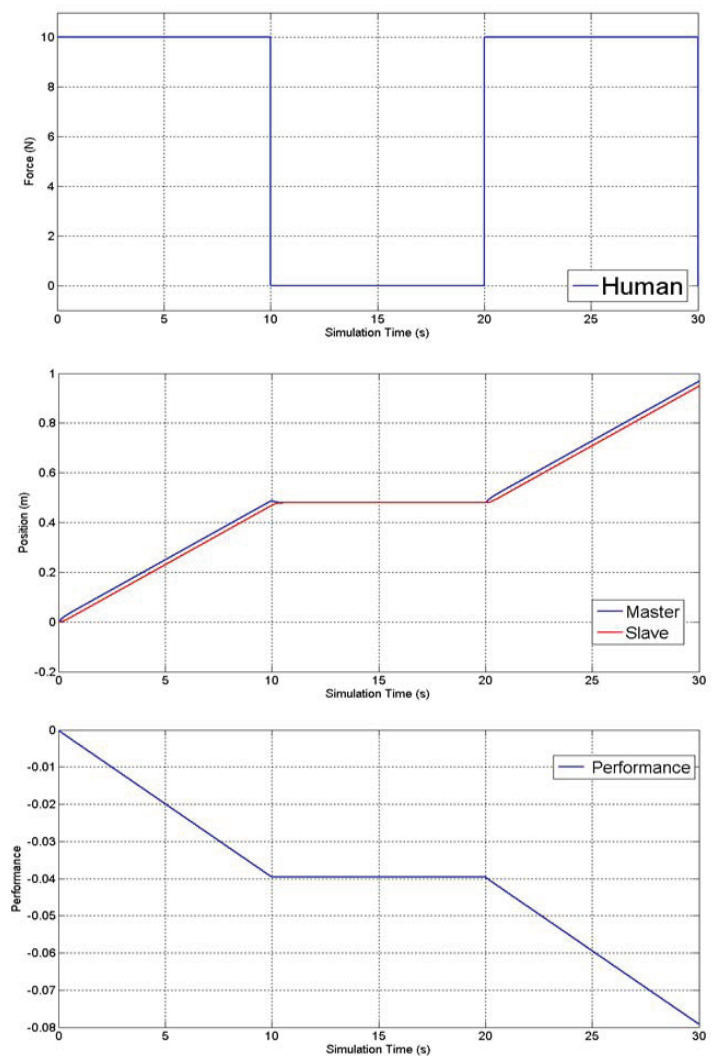

Fig. 2. Position Response in Abrupt Changing Motion

In order to simplify the system simulation, the master and slave models can be described as $m_{m} / s$ and $m_{s} / s$, where the effective endpoint mass $m_{m}=1 \mathrm{~kg}$ and $m_{s}=1 \mathrm{~kg}$,

$$
A=\left(\begin{array}{ccc}
0 & 0 & 0 \\
0 & 0 & 0 \\
1 & -1 & 0
\end{array}\right), \quad B=\left(\begin{array}{ll}
1 & 0 \\
0 & 1 \\
0 & 0
\end{array}\right)
$$


Besides, as mentioned above, $K_{0}$ is the local controller that does not consider the impact of network, the poles are given as $[-100.0]$, then,

$$
K_{0}=\left(\begin{array}{ccc}
100 & 0 & 0 \\
0 & 100 & 0
\end{array}\right)
$$

According to the theorems above, the global controllers $K_{1}$ and $K_{2}$ are obtained under $\gamma_{\min }=0.0066$, which is calculated by YALMIP/MATLAB,

$$
\begin{aligned}
& K_{1}=\left(\begin{array}{lll}
-1.4474 & 1.5123 & 242.3349
\end{array}\right) \\
& K_{2}=\left(\begin{array}{lll}
1.5699 & -1.6499 & -263.6271
\end{array}\right)
\end{aligned}
$$

For simulation purpose, the human operator is modeled as the pulse generator, as can be seen in upper Figure of Fig. 2. Middle Figure of Fig. 2 shows the tracking of position between the master and slave. It is clear that the position tracking is achieved in this case. Moreover lower Figure shows the value of the performance index $\int_{0}^{t}\left(z(s)^{T} z(s)-\gamma^{2} w(s)^{T} w(s)\right) d s<0$ in the simulation process. This index is always negative which induces that the prescribed performances are guaranteed. These three Figures show the effectiveness of this approach.

\section{B. Tracking in wall contact motion}

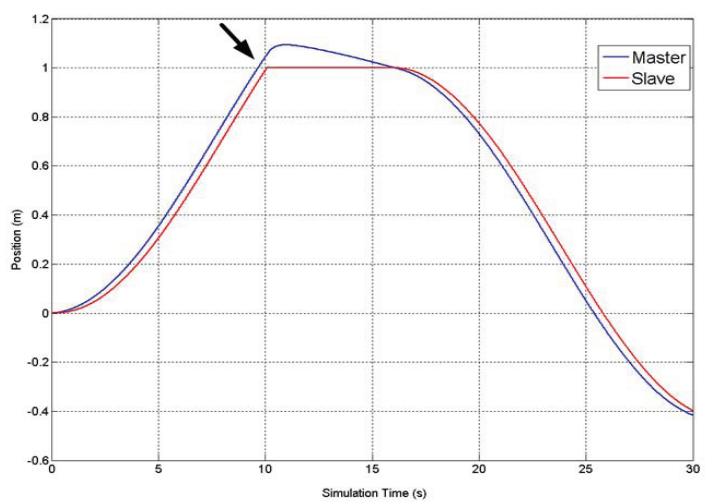

Fig. 3. Position Response in Wall Contact Motion

We also performed simulation in wall contact motion, where the slave is driven to the hard wall with a stiffness of $K_{e}=30 \mathrm{kN} / \mathrm{m}$ located at the position $x=1.0 \mathrm{~m}$. Like in abrupt change of motion, the system shows, in Fig. 3, a stable behavior with the global controllers, and the error that the arrow has marked between the master and the slave stays acceptable.

\section{CONCLUSIONS AND FUTURE WORKS}

We have addressed the study of teleoperation system, including the stability, and state synchronization. The main problem concerned in this paper is the time-varying delays in the communication line, with 2 channels. To solve this problem, novel system architecture has been proposed, based on the theory of Lyapunov-Krasovskii functional with $H_{\infty}$ control. Furthermore, on the basis of $H_{\infty}$ control, the paper realizes the high performance of the position tracking.

Numerical simulations, achieved by YALMIP/SIMULINK /MATLAB, have confirmed the accuracy of analysis, and proved that the teleoperation system, designed by our theory, Could run in different workshop conditions.

Future works will focus on expand the system class, for example, the proposed theory in the paper can be extended to nonlinear system, distributed system, and others. In this paper, we have used time-stamps to estimate time delays, in the future, the estimated delays could be eliminated, providing a memoryless controller. At last, some real implementations are being planned in our laboratory.

\section{REFERENCES}

[1] R.J. Anderson and M.W. Spong, Bilateral Control of Teleoperators with Time Delay, lEEE trans. on Automatic Control, vol. 34, 1989, pp 494-501.

[2] J. Cheong and S.-I. Niculescu, "Robust Motion Synchronization Control for Interconnected Systems with Human Interaction", in Proceedings of the 17th World Congress The International Federation of Automatic Control, Seoul, Korea, 2008, pp. 6347-6352.

[3] N. Chopra, M.W. Spong, R. Ortega and N.E. Barabanov, "On Position Tracking in Bilateral Teleoperation", in Proceeding of the 2004 American Control Conference, Boston, USA, 2004, pp. 5244-5249.

[4] E. Fridman, A New Lyapunov Technique For Robust Control of Systems with Uncertain Non-Small Delays, IMA Journal of Mathematical Control and Information, vol. 23, 2006, pp 165-179.

[5] E. Fridman, U. Shaked, New Bounded Real Lemma Representations for Time-Delay Systems and Their Applications, lEEE trans. on Automatic Control, vol. 46, 2001, pp 1973-1979.

[6] L.G. Garcia-Valdovinos, V. Parra-Vega and M.A. Arteaga, "ObserverBased Higher-Order Sliding Mode Impedance Control of Bilateral Teleoperation under Constant Unknown Time Delay", in Proceedings of the 2006 IEEE/RSJ International Conference on Intelligent Robots and Systems, Beijing, China, 2006, pp. 1692-1699.

[7] K. Gu, V.L. Kharitonov and J. Chen, Stability of Time-Delay Systems, Boston, Basel, Berlin, 2003.

[8] Y. He, M. Wu, J.-H. She and G.-P. Liu, Parameter-Dependent Lyapunov Functional for Stability of Time-Delay Systems with PolytopicType Uncertainties, lEEE trans. on Automatic Control, vol. 49, 2002, pp 828-832.

[9] W.-J. Jiang, A. Kruszewski, J.-P. Richard and A. Toguyeni, "A Gain Scheduling Strategy for the Control and Estimation of A Remote Robot via Internet", in The 27th Chinese Control Conference, Kunming, China, 2008, pp. 793-799.

[10] G. Niemeyer and J.-J.E. Slotine, Stable Adaptive Teleoperation, IEEE Journal of Oceanic Engineering, vol. 16, 1991, pp 152-162.

[11] J.-P. Richard, Time Delay Systems: an Overview of Some Recent Advances and Open Problems, Automatica, vol. 39, 2004, pp 16671694.

[12] J.-H. Ryu, D.-S. Kwon and B. Hannaford, Stable Teleoperation with Time-Domain Passivity Control, lEEE trans. on Robotics and Automation, vol. 20, 2004, pp 365-373.

[13] G. Tadmor, The Standard $H_{\infty}$ Problem in Systems with A Single Input Delay, lEEE trans. on Automatic Control, vol. 45, 2000, pp 382-397.

[14] S.-Y. Xua, J. Lam and Y. Zou, New Results on Delay-Dependent Robust $H_{\infty}$ Control for Systems with Time-Varying Delays, Automatica, vol. 42, 2006, pp 343-348.

[15] S. Zampieri, "Trends in Networked Control Systems", in Proceedings of the 17th World Congress The International Federation of Automatic Control, Seoul, Korea, 2008, pp. 2886-2894.

[16] X.-M. Zhang and Q.-L. Han, Robust $H_{\infty}$ Filtering for A Class of Uncertain Linear Systems with Time-Varying Delay, Automatica, vol. 44, 2008, pp 157-166. 